\title{
A dissociative fluorescence enhancement technique for one-step time-resolved immunoassays
}

\author{
Kaj R. Blomberg • Veli-Matti Mukkala • \\ Harri H. O. Hakala • Pauliina H. Mäkinen • \\ Mikko U. Suonpää • Ilkka A. Hemmilä
}

Received: 6 October 2010 /Revised: 19 November 2010 /Accepted: 26 November 2010 /Published online: 15 December 2010

(C) The Author(s) 2010. This article is published with open access at Springerlink.com

\begin{abstract}
The limitation of current dissociative fluorescence enhancement techniques is that the lanthanide chelate structures used as molecular probes are not stable enough in one-step assays with high concentrations of complexones or metal ions in the reaction mixture since these substances interfere with lanthanide chelate conjugated to the detector molecule. Lanthanide chelates of diethylenetriaminepentaacetic acid (DTPA) are extremely stable, and we used EuDTPA derivatives conjugated to antibodies as tracers in one-step immunoassays containing high concentrations of complexones or metal ions. Enhancement solutions based on different $\beta$-diketones were developed and tested for their fluorescence-enhancing capability in immunoassays with EuDTPA-labelled antibodies. Characteristics tested were fluorescence intensity, analytical sensitivity, kinetics of complex formation and signal stability. Formation of fluorescent complexes is fast $(5 \mathrm{~min})$ in the presented enhancement solution with EuDTPA probes withstanding strong complexones (ethylenediaminetetra acetate (EDTA) up to $100 \mathrm{mM}$ ) or metal ions (up to $200 \mu \mathrm{M}$ ) in the reaction mixture, the signal is intensive, stable for $4 \mathrm{~h}$ and the analytical sensitivity with $\mathrm{Eu}$ is $40 \mathrm{fmol} / \mathrm{L}, \mathrm{Tb} 130 \mathrm{fmol} / \mathrm{L}$, $\mathrm{Sm} 2.1 \mathrm{pmol} / \mathrm{L}$ and Dy $8.5 \mathrm{pmol} / \mathrm{L}$. With the improved fluorescence enhancement technique, EDTA and citrate plasma samples as well as samples containing relatively high concentrations of metal ions can be analysed using a one-step immunoassay format also at elevated temperatures. It facilitates four-plexing, is based on one chelate structure for detector molecule labelling and is suitable for
\end{abstract}

K. R. Blomberg $(\square) \cdot$ V.-M. Mukkala $\cdot$ H. H. O. Hakala

P. H. Mäkinen · M. U. Suonpää · I. A. Hemmilä

PerkinElmer Inc, Wallac Oy,

P.O. Box 10, 20101 Turku, Finland

e-mail: kaj.blomberg@perkinelmer.com immunoassays due to the wide dynamic range and the analytical sensitivity.

Keywords Time-resolved fluorometry · Dissociative fluorescence enhancement · Immunoassay · Multiplexing · $\mathrm{Eu} \cdot \mathrm{Sm} \cdot \mathrm{Tb} \cdot \mathrm{Dy} \cdot$ Antibody

\section{Introduction}

Ions of the lanthanides $\mathrm{Eu}, \mathrm{Sm}, \mathrm{Tb}$ and to a lesser degree Dy have successfully been exploited as labels in bioanalytical assay for the last two decades [1-3]. Of all the different ways to apply lanthanide labels to bioanalytical assays, the most common technique is the one which is based on nonfluorescent lanthanide chelates $[4,5]$. In this technique, the ion is chelated to polyaminopolyacetic acids which in turn are conjugated to the detector molecule. When the bioaffinity reaction is completed, the lanthanide ions are dissociated from the chelates on the detector molecule, into a fluorescence-enhancing solution containing ligands that form a fluorescent metal complex with the ions. Due to a long fluorescence decay time and intensive fluorescence, time-resolved fluorometry is the detection method of choice, i.e. the fluorescence is measured after the background fluorescence has decayed. In this way, the autofluorescence can be omitted resulting in ultimate analytical sensitivity $[4,6]$. This technique has been widely employed in immunoassays, nucleic acid hybridisation assays, receptor-ligand assays, enzyme activity measurements and in various other applications $[1,6-8]$. The physicochemical properties of different enhancement solutions and chelates have been discussed in several papers [5, 9, 10-12].

The lanthanide chelates attached to the detector molecule must be stable enough to withstand the 
conditions in the reaction mixture without dissociation of the lanthanide ion from the chelate. On the other hand, the ion should be rapidly dissociated into the enhancement solution before the fluorescence measurement; otherwise, the technique is not fast enough for routine laboratory applications and automation. Commercially available dissociated-enhanced lanthanide fluorescent immunoassay (DELFIA ${ }^{\circledR}$ ) chelates and enhancement solutions meet these criteria in that serum and plasma samples not containing strong complexones can be used whereas samples containing high concentrations of complexones or metal ions should be avoided in onestep assays. This is sometimes a limitation in the clinical laboratory as blood is taken into both serum and plasma tubes and thus there is a need for a technology that works with all types of samples [13].

The aim of this study was to develop an enhancement solution that quickly and efficiently dissociates lanthanide ions from chelates that are stable at harsh assay condition involving strong complexones and heavy metals. One-step assays with high concentrations of citrate, ethylenediaminetetra acetate (EDTA) or metal ions, require that the detector molecule is labelled with an extremely stable lanthanide chelate. Such assays are immunoassays based on citrate and EDTA plasma samples, enzyme activity assays relying on high concentrations of metal ions in order for the enzyme to be active, soil analysis with immunoassays, cytotoxicity assays and nucleic acid assays carried out partly at relatively high temperatures $[7-10,14]$.

\section{Materials and methods}

\section{Reagents and buffers}

Ten $\beta$-diketone molecules of the formula $\mathrm{R}_{1}-\mathrm{C}(=\mathrm{O})-\mathrm{CH}_{2}-$ $C(=O)-R_{2}$ where $R_{1}$ was a naphthyl, benzofuryl, furyl or thienyl group and $\mathrm{R}_{2}$ was an alkyl chain with two or three carbon atoms substituted with five to seven fluorine atoms, tri- $n$-octylphosphineoxide (TOPO), Eu (100 nmol/L), Sm $(10 \mu \mathrm{mol} / \mathrm{L}), \mathrm{Tb}(100 \mu \mathrm{nmol} / \mathrm{L})$ and Dy $(10 \mu \mathrm{mol} / \mathrm{L})$ standard solutions, isothiocyanate-activated diethylenetriaminepentaacetic acid (DTPA) lanthanide chelates, DELFIA $^{\circledR}$ clear microtitration strip plates and DELFIA enhancement solution were obtained from PerkinElmer Inc. Anti-human chorionic gonadotropin beta unit $(\mathrm{hCG} \beta)$ antibody 2,764 , alpha-fetoprotein (AFP) antibody IF5 and assay buffer, washing solution and hCG $\beta$ and AFP-coated cups and calibrators were from the DELFIA Xpress Free hCG $\beta$ and AFP Immunoassay Kits (PerkinElmer). EDTA, $\mathrm{CuSO}_{4}$ and Triton X-100 were purchased from Aldrich.

Characterisation of the enhancement solutions

Fluorescence characteristics of the synthesised $\beta$-diketones with Eu were tested in a glycine buffer at $\mathrm{pH} 2.3$ containing various concentrations of the water protecting agent TOPO $(25-120 \mu \mathrm{M})$, detergent Triton X-100 $(0.05-0.2 \% v / v)$ and the $\beta$-diketone $(1-100 \mu \mathrm{M})$ to be tested. The concentrations of the components of the enhancement solutions were primarily optimised with respect to fluorescence intensity of $\mathrm{Eu}$, fluorescence signal stability, dissociation kinetics of EuDTPA chelates and $\mathrm{Eu}(\beta \text {-diketone })_{3}$ complex formation velocity. Fluorescence intensity and analytical sensitivity were determined by first diluting one volume of $\mathrm{Eu}$ standard with nine volumes of the enhancement solution to be tested, and then the obtained solution were serially diluted with the enhancement solution. The fluorescence from $200 \mu \mathrm{l}$ of the serial dilutions of Eu standards was measured in 96-well microtitration strip plates with a timeresolved fluorometer (Victor D, PerkinElmer). Standard dilutions of $\mathrm{Sm}, \mathrm{Tb}$ and Dy were prepared and tested as described for $\mathrm{Eu}$ in the enhancement solution with the most intensive $\mathrm{Eu}$ fluorescence. The fluorescence from $\mathrm{Tb}$ and Dy were measured after addition of $50 \mu \mathrm{l}$ of dipicolinic acid derivative (DELFIA Enhancer, PerkinElmer) to the serially diluted standards. Instrument settings used are shown in Table 1. Excitation and emission spectra for the

Table 1 Fluorescence of Eu and Sm in the BFPP-based enhancement solution

\begin{tabular}{|c|c|c|c|c|c|c|c|c|c|}
\hline \multicolumn{6}{|c|}{ Fluorometer settings } & \multicolumn{2}{|c|}{ Fluorescence $(n=4)$} & \multicolumn{2}{|l|}{ Limit of blank } \\
\hline Lanthanide & $\begin{array}{l}\text { Excitation } \\
\text { filter }(\mathrm{nm})\end{array}$ & $\begin{array}{l}\text { Emission } \\
\text { filter }(\mathrm{nm})\end{array}$ & $\begin{array}{l}\text { Delay } \\
\text { time }(\mu \mathrm{s})\end{array}$ & $\begin{array}{l}\text { Window } \\
\text { time }(\mu \mathrm{s})\end{array}$ & $\begin{array}{l}\text { Cycle } \\
\text { time }(\mu \mathrm{s})\end{array}$ & $\begin{array}{l}\text { Counts at } \\
1 \mathrm{nmol} / \mathrm{L}\end{array}$ & $\begin{array}{l}\text { Background } \\
\text { Signal }\end{array}$ & $\begin{array}{l}\text { Concentration } \\
(\mathrm{fmol} / \mathrm{L})\end{array}$ & $95 \% \mathrm{CI}$ \\
\hline $\mathrm{Eu}$ & 340 & 615 & 400 & 400 & 1,000 & $1,112,000$ & 113 & 40 & $30-64$ \\
\hline $\mathrm{Sm}$ & 340 & 642 & 50 & 100 & 1,000 & 6,092 & 62 & 2,100 & $1,610-3,370$ \\
\hline $\mathrm{Tb}$ & 340 & 545 & 500 & 1,400 & 2,000 & 571,300 & 825 & 130 & $86-181$ \\
\hline Dy & 340 & 572 & 30 & 30 & 1,000 & 8,824 & 350 & 8,500 & $6,310-13,200$ \\
\hline
\end{tabular}

$\mathrm{Tb}$ and Dy were measured after addition of dipicolinic acid 
lanthanides in the developed enhancement solutions were measured with a PerkinElmer LS5 fluorescence spectrometer. The velocity of fluorescent complex formation and the stability of the fluorescence signal in the enhancement solutions were determined by adding $5 \mu \mathrm{L}$ of EuDTPAlabelled AFP antibody and 195- $\mu \mathrm{L}$ enhancement solution into microtitration plate wells that were shaken for $5 \mathrm{~min}$ at room temperature and measuring the fluorescence as a function of time. Enhancement solutions were stored in dark at $4{ }^{\circ} \mathrm{C}$ and the fluorescence from $1 \mathrm{nmol} / \mathrm{L}$ of $\mathrm{Eu}$ in the enhancement solution was measured at various time points as described above.

\section{Antibody labelling}

Detector antibodies in these two assays were labelled with europium chelate of 1-(4-isothiocyanatobenzyl) diethylenetriamine- $N, N, N^{\prime}, N^{\prime \prime}, N^{\prime \prime}$-pentaacetic acid. The antibody at a concentration of $5 \mathrm{mg} / \mathrm{mL}$ was incubated with a 55 -fold molar excess of the chelate in $50 \mathrm{mM}$ carbonate buffer $\mathrm{pH} 9.5$ over night at $4{ }^{\circ} \mathrm{C}$. Labelled antibody was separated from unreacted chelates by gel filtration (Superdex 200, 1.6 $\times 60 \mathrm{~cm}$, Pharmacia) with a buffer containing $50 \mathrm{mmol} / \mathrm{L}$ Tris-HCl, $9 \mathrm{~g} / \mathrm{L} \mathrm{NaCl}, \mathrm{pH} 7.8$, as eluation buffer. When the ratio $\mathrm{Eu} / \mathrm{IgG}$ was determined by measuring the Eu concentration of conjugated antibody against an Eu standard and the protein concentration with absorbance, a conjugation level of ten was achieved. The principle of protein labelling with bifunctional chelating agents and coating of microtitration plates have in great detail been described elsewhere [7].

\section{Immunoassays}

Blood samples were taken from volunteers into plain tubes and EDTA-anticoagulant tubes. Informed consent was obtained from all volunteers and the samples were deidentified to ensure privacy. The study site has obtained approval to conduct studies for research purpose from the local ethical committee. The samples were measured in duplicate on a DELFIAXpress ${ }^{\circledR}$ immunoanalyzer (PerkinElmer). In the DELFIA, Xpress analyzer sample $(10 \mu \mathrm{L})$ and assay buffer $(25 \mu \mathrm{L})$ are pipetted simultaneously into anti-analyte antibody coated cups. In $5 \mu \mathrm{L}, 50 \mathrm{ng}$ of Eu-labelled detector antibody is added and the cups are incubated with shaking at $+35{ }^{\circ} \mathrm{C}$ for 20 min after which they are washed four times. The fluorescence from the cups is measured after $100 \mu \mathrm{L}$ of the developed enhancement solution was dispensed and the cups incubated for $5 \mathrm{~min}$ at $+35^{\circ} \mathrm{C}$. Antibody bound $\mathrm{Eu}$ is dissociated from DTPA into the enhancement solution in which it forms intensively fluorescent complexes with the $\beta$-diketone. The effect of the complexone
EDTA and heavy metal $\mathrm{Cu}^{2+}$ on the stability of the detector molecule label EuDTPA was also tested by adding EDTA or $\mathrm{Cu}$ at various concentrations to the assay buffer in order to get total concentrations in the assay mixture ranging from $1-200 \mathrm{mmol} / \mathrm{L}$ of EDTA or $0-1,000 \mu \mathrm{mol} / \mathrm{L}$ of $\mathrm{Cu}$. The effect was tested with the analyzer at three different hCG $\beta$ concentrations in duplicate, 236, 112 and $22 \mathrm{ng} / \mathrm{mL}$.

\section{Results}

Fluorescence intensity and signal stability

Of all the $\beta$-diketones tested, the structure 1-(2-benzofuryl)4,4,5,5,5-pentafluoro-1,3-pentanedione (BFPP) had the most favourable properties with respect to fluorescence intensity, analytical sensitivity, complex formation kinetics and signal stability in a glycine buffer at $\mathrm{pH} 2.3$ with $50 \mu \mathrm{M}$ TOPO and $0.2 \%(v / v)$ Triton $\mathrm{X}-100$. This enhancement solution dissociates the lanthanide ions from LnDTPA and forms in $5 \mathrm{~min}$ an intensively fluorescing complex between BFPP and lanthanide. Once the $\operatorname{Ln}(\mathrm{BFPP})_{3}$ complex is formed, the signal is stable for several hours in the solution (Table 2.) and the enhancement solution itself is stable upon storage at $+4{ }^{\circ} \mathrm{C}$ for at least 18 months (Table 3 ).

\section{Spectra}

Excitation and emission spectra for the lanthanides are depicted in Fig. 1. All four lanthanides emit at one principal wavelength and the emission peaks are narrow and well separated from each other.

Limit of blank and dynamic range

The limit of blank (mean+3SD with 95\% CI) for $\mathrm{Eu}$ in developed enhancement solution, 40 (30-64) fmol/L, is of the same order of magnitude than that of DELFIA enhancement solution, 80 (115-200) fmol/L. The lowest

Table 2 Kinetics of fluorescent complex formation in the BFPPbased fluorescence enhancement solution at RT

\begin{tabular}{lcc}
\hline Incubation time (min) & Fluorescence (counts, $\left.10^{3}\right)$ & $\mathrm{CV} \%(n=3)$ \\
\hline 5 & 1,800 & 4.5 \\
15 & 1,850 & 4.2 \\
30 & 1,853 & 4.0 \\
60 & 1,858 & 4.4 \\
120 & 1,869 & 4.7 \\
240 & 1,849 & 4.7 \\
\hline
\end{tabular}


Table 3 Storage stability of the BFPP-based enhancement solution at $+4{ }^{\circ} \mathrm{C}$

\begin{tabular}{lcc}
\hline Storage time (days) & Fluorescence (counts, $\left.10^{3}\right)$ & CV\% $(n=6)$ \\
\hline 0 & 1,252 & 0.9 \\
6 & 1,316 & 1.9 \\
13 & 1,256 & 1.1 \\
27 & 1,237 & 0.9 \\
41 & 1,263 & 1.1 \\
83 & 1,263 & 0.3 \\
178 & 1,259 & 0.2 \\
345 & 1,158 & 0.3 \\
539 & 1,267 & 0.6 \\
\hline
\end{tabular}

limit of blank is achieved with Eu followed by $\mathrm{Tb}, \mathrm{Sm}$ and Dy (Table 1). In the developed enhancement solution the relationship between the Ln concentration and the fluorescent signal is linear up to $10 \mathrm{nmol} / \mathrm{L}$ of $\mathrm{La}$ and the fluorometer is linear up to $5 \times 10^{6}$ fluorescence counts with the instrument settings shown in Table 1 . These characteristics result in a dynamic range that is about $5 \operatorname{logs}$ for $\mathrm{Eu}$ and $\mathrm{Tb}$ and 4 logs for Sm and Dy, respectively.

Effect of edta plasma and metal ions

In immunoassays of $h C G \beta$ and AFP the regression equations show no difference in the measured concentration levels between serum and EDTA plasma samples, indicating that the EuDTPA chelate conjugated to the detector

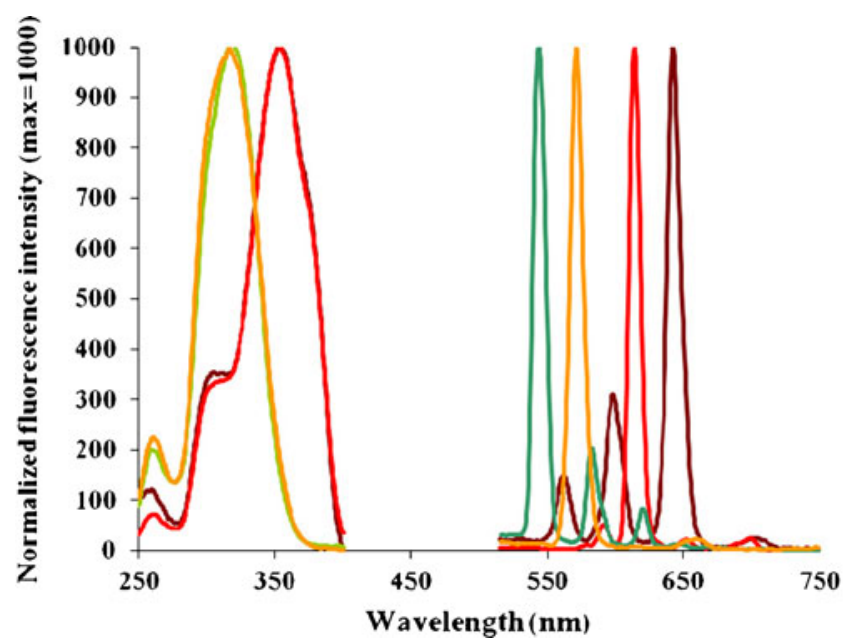

Fig. 1 Excitation and emission spectra for $\mathrm{Eu}, \mathrm{Sm}, \mathrm{Tb}$ and Dy. The spectra for Eu (red) and Sm (dark red) were measured in the BFPPbased enhancement solution. Excitation maxima, emission maxima and decay times obtained were $354 \mathrm{~nm}, 614 \mathrm{~nm}$ and $570 \mu$ s for $\mathrm{Eu}$ and $353 \mathrm{~nm}, 643 \mathrm{~nm}$ and $43 \mu$ s for Sm, respectively. The spectra for Tb (green) and Dy (orange) were determined after addition of dipicolinic acid to the BFPP enhancement solution. Excitation maxima, emission maxima and decay times for Tb were $319 \mathrm{~nm}, 544 \mathrm{~nm}$ and 1,190 $\mathrm{ss}$ and for Dy $317 \mathrm{~nm}, 572 \mathrm{~nm}$ and $20 \mu$ s, respectively antibody is stable and withstands the chelating effect of EDTA in plasma samples (Fig. 2). When EDTA was added to the reaction mixture in an assay of serum $h C G \beta$ the signal is stable up to $100 \mathrm{mmol} / \mathrm{L}$ of EDTA for all three hCG $\beta$ concentration levels measured. In an assay of hCG $\beta$ in serum samples, the measured concentrations are unchanged with up to $200 \mu \mathrm{mol} / \mathrm{L} \mathrm{C} \mathrm{Cu^{2+ }}$ present at three different hCG $\beta$ concentrations (Fig. 3). The criterion for stability was the $P$ value for the slopes from the regression analysis of EDTA or $\mathrm{Cu}^{2+}$ concentrations present vs. the response in the hCG $\beta$ assay for all three $\mathrm{hCG} \beta$ concentrations tested. We concluded that if $P>0.05$, the slopes were not significantly different from zero, and hence there were no evidence for dissociation of Eu from the labelled antibody.

\section{Discussion}

In dissociative fluorescence enhancement techniques relying on lanthanide probes, the fluorescence is measured in an enhancement solution after completion of the biospecific reaction. Immunoassays using this technique are usually performed on microtitration plates in which bound and free tracer is separated by washing and the fluorescence of the bound fraction (solid phase) is measured after addition of enhancement solution to the wells of the plates. An enhancement solution useful for routine laboratory applications is characterized by an intensive fluorescence (absorptivity $\times$ quantum yield), wide dynamic range, rapid dissociation in the enhancement solution of the lanthanide ions from the chelates that are conjugated to detector molecules, fast formation of fluorescent $\mathrm{Ln}$ complexes with the components of the enhancement solution, a stable fluorescence signal and long storage stability.

The enhancement solution and diethylenetriaminetetra acetate (DTTA) chelates used in the DELFIA technology meet these criteria to a high degree. In the enhancement solution of this technology, lanthanide ions rapidly dissociate from the DTTA chelates due low pH (3.2). Eu and Sm form a fluorescent complex with the light absorbing trifluorinated $\beta$-diketone 2-naphtoyltrifluoroacetone (2NTA). Lanthanide chelates of DTTA are stable in a reaction mixture not containing strong complexones such as EDTA and dissociate and form highly fluorescent 2-NTA complexes in less than $5 \mathrm{~min}$ in the enhancement solution.

In immunoassays intended for the clinical laboratory the main limitation of the present DELFIA technique is that citrate and EDTA plasma samples cannot be used in onestep assay formats since Ln ions may dissociate from DTTA at these conditions.

In this study the goal was to develop a variant of the dissociative enhancement technique that is robust and fast 
Fig. 2 Linear regression analysis of serum and EDTA plasma samples analysed for hCG $\beta$ and AFP using a one-step assay format. Concentration level difference between the two sample types was not observed. a hCG $\beta$ assay, $y=1.001 \times-0.16 ; y / x=$ $0.329 ; n=22 ; r=0.998$. b AFP assay, $y=0.958 \times+0.611 ; y / x=$ $5.990 ; n=26 ; r=0.996$

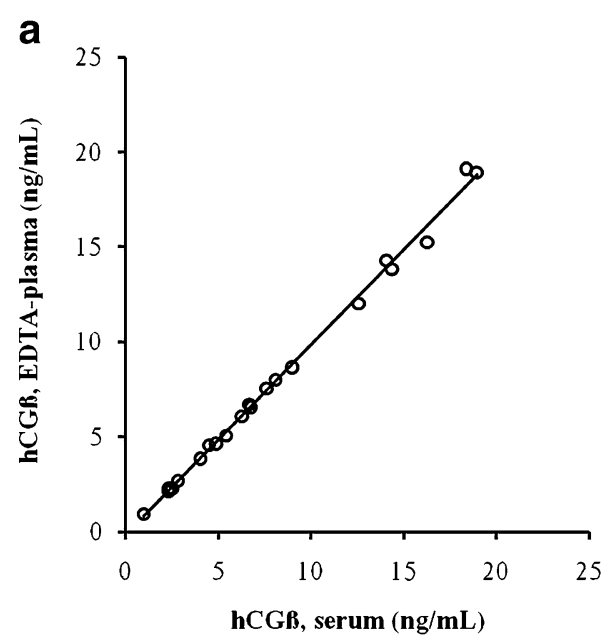

enough with samples containing complexones and metal ions at concentrations interfering with LnDTTA chelates. The interference from complexones and metal ions in the biospecific reaction mixture can be avoided by replacing

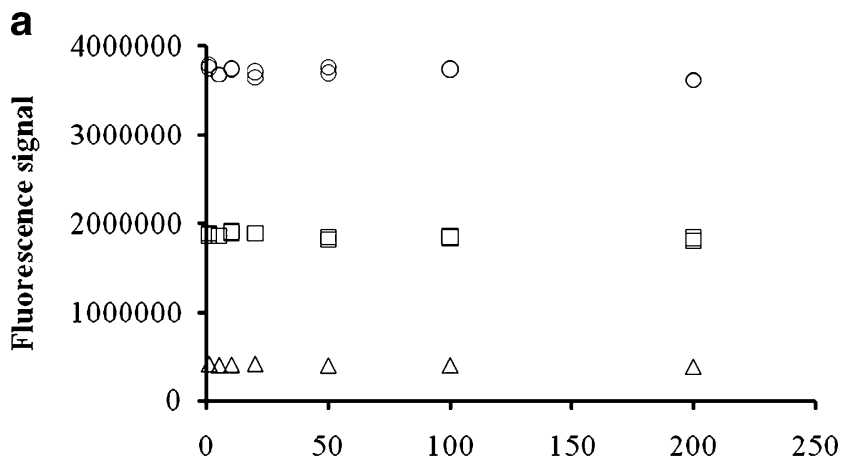

EDTA Concentration $(\mathbf{m m o l} / \mathbf{L})$

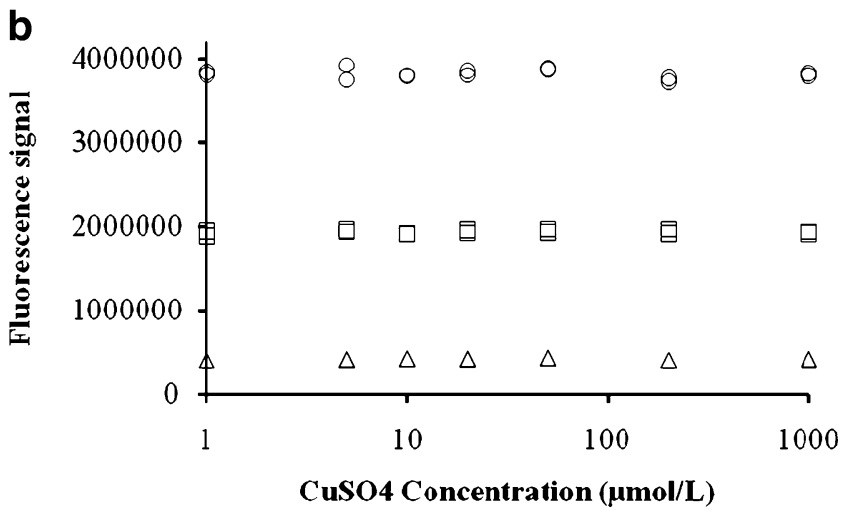

Fig. 3 Effect of EDTA and $\mathrm{Cu}^{2+}$ on the stability of the EuDTPA derivative used for detector antibody labelling in the hCG $\beta$ assay. a The $P$ value was $>0.05$ for up to $100 \mathrm{mmol} / \mathrm{L}$ EDTA for all three hCG $\beta$ concentrations tested (from the highest to the lowest signal level, $P=0.68$ (circles); $P=0.07$ (squares); $P=0.67$ (triangles)). b The $P$ value was $>0.05$ for up to $200 \mu \mathrm{mol} / \mathrm{L} \mathrm{Cu}^{2+}$ for all three hCG $\beta$ concentrations tested (from the highest to the lowest signal level, $P=$ 0.11 (circles); $P=0.27$ (squares); $P=0.42$ (triangles))

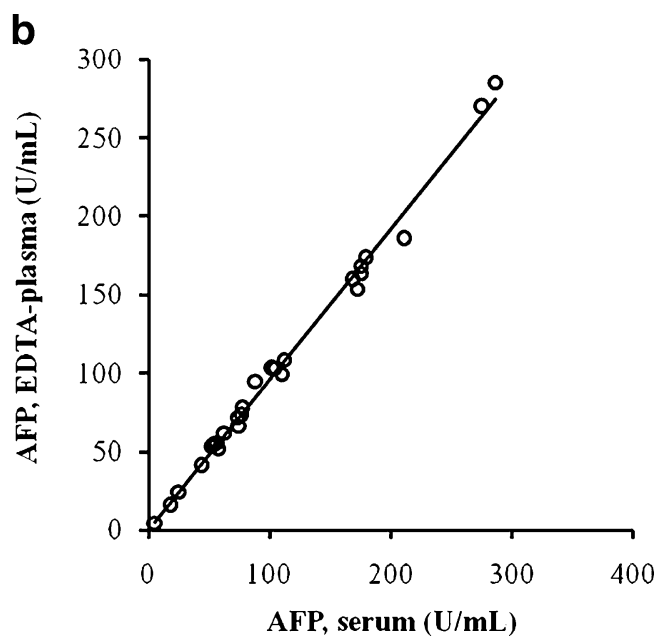

LnDTTA with chelates possessing a higher stability. DTPA chelates of Ln withstand the citrate and EDTA in plasma samples due to a high stability constant $\left(K=10^{23}\right)$ [15], but the drawback is the slow dissociation at $\mathrm{pH}>3.0(120 \mathrm{~min})$ [16], the lowest $\mathrm{pH}$ at which for instance 2-NTA-based enhancements work. The complex formation between Ln ions and 2-NTA is incomplete at lower $\mathrm{pH}$. A long dissociation and complex formation time is unpractical in the laboratory and makes automation difficult. Therefore, a 2-NTA-based enhancement solution together with LnDTPA conjugates is not the best of choices.

A number of potential $\beta$-diketones were screened for their complex formation ability with $\mathrm{Ln}$ at $\mathrm{pH}$ values below 3 . Acidic conditions increase the dissociation of LnDTPA chelates as the equilibrium is towards protonated DTPA and an excess of $\beta$-diketone further shifts the equilibrium towards Ln- $\beta$-diketone complexes The $\beta$-diketones investigated have three essential features: a light absorbing aryl group, two ketone groups that binds to the coordination sites of the Ln ion and a fluorinated alkyl group that shifts the ketone-enole equilibrium towards the enole form. Five or seven strongly electronegative fluorine atoms make these $\beta$-diketones more acidic than 2-NTA with three fluorine atoms. These properties ensure efficient absorption of light upon excitation and complex formation at low $\mathrm{pH}$. Of all the candidate $\beta$ diketones tested, the characteristics of BFPP met the requirements best. The benzofuryl group absorbs excitation light efficiently and the pentafluorinated alkyl group shifts the equilibrium towards the enole form also at low $\mathrm{pH}$, which facilitates complex formation with Eu. The developed enhancement solution was primarily optimised for $\mathrm{Eu}$ detection and contains BFPP, TOPO and Triton X-100 in a glycine buffer at $\mathrm{pH} 2.3$. Also, Sm fluoresces strongly in this solution. The synergistic agent TOPO and the micelleforming detergent Triton X-100 further protects the Ln $(\mathrm{BFPP})_{3}$ complex from fluorescence quenching water molecules by replacing them at remaining coordination sites in 
the complex and by solubilizing the complexes in micelles. LnDTPA chelates dissociate rapidly in this solution. Dipicolinic acid derivative at neutral $\mathrm{pH}$ is a better fluorescenceenhancing ligand than BFPP for $\mathrm{Tb}$ and Dy because of its higher excited triplet state energy level. In quadruple label assays $\mathrm{Eu}$ and $\mathrm{Sm}$ are measured in the BFPP-based enhancement solution subsequent to the completion of the biospecific reaction and the fluorescence of the dissociated $\mathrm{Tb}$ and Dy are detected after addition of a dipicolinic acid derivative containing solution that raises the $\mathrm{pH}$ to neutral.

The fluorescence intensities of Eu and $\mathrm{Sm}$ in the developed enhancement solution as well as the achieved analytical sensitivities compare well with the established 2-NTA-based enhancement solutions. Tb and Dy can be detected after addition of dipicolinic acid. The dynamic range for $\mathrm{Eu}$ and $\mathrm{Tb}$ is about 5 logs and for Sm and Dy 4 logs, which is identical to the dynamic range for 2-NTA-based DELFIA enhancement solution. Most of the fluorescence is emitted at one wavelength as a narrow peak and all four lanthanides emit at different wavelengths. They can be distinguisted from each other, due to the favourable spectra (spectral resolution) and by utilising their different fluorescence decay times for temporal resolution i.e. employing different delay times when measuring the lanthanides. This permits quadruple-plex assays to be designed, although overlapping spectra must be accounted for and corrected for when detecting Sm in wells with high Eu concentrations and Dy in prescence of high $\mathrm{Tb}$ concentrations. The assay requiring the lowest limit of detection should be labelled with $\mathrm{Eu}$, followed by $\mathrm{Tb}, \mathrm{Sm}$ and Dy. Usually up to 20 DTPA chelates can be conjugated to an antibody without any severe effects on the affinity which makes it easy to set up sandwich immunoassays that work in the picomolar range. In the assays for hCG $\beta$ and AFP the detector antibodies were labelled with an isothiocyanate derivative of DTPA which reacts with amino groups. SH and $\mathrm{COO}$ groups can be labelled with iodoacetamido and amino derivatives of DTPA, respectively. There is no dissociation of $\mathrm{Eu}$ from DTPA at $35^{\circ} \mathrm{C}$ in an assay mixture with human serum containing EDTA, nor is any dissociation observed with $200 \mu \mathrm{M}$ of $\mathrm{Cu}^{2+}$ present, showing that this enhancement technique can be used in one-step assays containing complexones or metal ions, and at physiological temperatures.

The presented fluorescence enhancement technique for bioanalytical assays offers advantages over current techniques, such as, one-step assays with EDTA and other plasma samples containing complexones as anti-coagulants, lanthanide labelling chelate stability at high concentrations of metal ions and at elevated temperatures, important in some DNA hybridisation assays as well as some enzymatic assays and rapid immunoassays. Four response parameters can be measured from the same assay mixture with the presented technique. The lanthanides are chelated to DTPA which is stable at a vast range of assay condition resulting in that one chelate structure works for almost all assay designs and applications. Automation is easier with the improved fluorescence enhancement technique due to fewer steps and hence shorter assay protocols.

Open Access This article is distributed under the terms of the Creative Commons Attribution Noncommercial License which permits any noncommercial use, distribution, and reproduction in any medium, provided the original author(s) and source are credited.

\section{References}

1. Hemmilä IA (1991) Applications of fluorescence in immunoassays. Wiley, New York

2. Christopoulos TK, Diamandis EP (1996) In: Diamandis EP, Chrostopoulos TK (eds) Immunoassay. Academic, London

3. Hemmilä I, Mukkala V-M (2001) Time-resolution in fluorometry, technologies, labels, and applications in bioanalytical assays. Crit Rew Clin Lab Sci 38:441-519

4. Soini E, Hemmilä I (1979) Fluoroimmunoassays: present status and key problems. Clin Chem 25:353-361

5. Hemmilä I, Dakubu S, Mukkala V-M, Siitari H, Lövgren T (1984) Europium as a label in time-resolved immunofluorometric assays. Anal Biochem 137:335-343

6. Hemmilä I, Ståhlberg T, Mottram P (1995) Bioanalytical applications of labelling technologies, 2nd edn. Wallac Oy, Turku

7. Hemmilä I (1997) In: Johnston AP, Turner MW (eds) Immunochemistry, practical approach series. IRL Press of Oxford University Press, Oxford

8. Hemmilä I (1997) In: Devlin JP (ed) High throughput screening. Marcel Dekker, New York

9. Hemmilä I (1985) Time-resolved fluorometric determination of terbium in aqueous solution. Anal Chem 57:1676-1681

10. Hemmilä I, Mukkala V-M, Latva M, Kiilholma P (1993) Di- and tetracarboxylate derivatives of pyridines, bipyridines and terpyridines as lumonigenic reagents for time-resolved fluorometric determination of terbium and dysprosium. J Biochem Biophys Meth 26:283-290

11. Degan P, Abbondandolo A, Montanogli G (1990) A new fluorescence enhancement solution for europium-based timeresolved fluoroimmunoassays. J Lumin Chemilumin 5:207-212

12. Yang J-H, Zhu G-Y, Wu B (1987) Enanced luminiscence of the europium/terebium/theonyltrifluoroacetone/1, 10-phenantroline/ surfactant system. Anal Chim Acta 198:287-292

13. Holtcamp U, Klein J, Sander J, Peter M, Janzen N, Steuerwald U, Blankenstein U (2008) EDTA in dry blood spots leads to false results in neonatal endocrinologic screening. ClinChem 54:602605

14. Blomberg K, Granberg C, Hemmilä I, Lövgren T (1986) Europium-labelled target cells in an assay of natural killer cell activity. J Immunol Meth 86:225-229

15. Möller T, Martin DF, Thompson LC, Ferrus R, Feistel GR, Randall WJ (1965) The coordination chemistry of yttrium and the rare earth metal ions. Chem Rev 65:1-50

16. Mukkala V-M, Mikola H, Hemmilä I (1989) The synthesis and use of activated $\mathrm{N}$-benzyl derivatives of diethylenetriaminetetraacetic acids: alternative reagents for labeling of antibodies with metal ions. Anal Biochem 176:319-325 